\title{
Effect of Cycled Feeding by Diets Including Vegetable and Fish Oil on Growth Performances and Fatty Acid Profiles of Rainbow Trout (Oncorhynchus mykiss Walbaum, 1792)
}

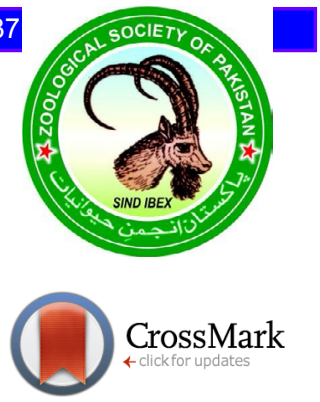

\author{
Seval Dernekbasi* and Emin Karatas \\ Univesity of Sinop, Faculty of Fisheries and Aquaculture, Department of Aquaculture, \\ Sinop, Turkey
}

\begin{abstract}
A B S T RA C T
The aim of this study was to evaluate the effects of alternate cycled feeding regime with diets including different oil sources on fatty acid profiles and growth performances of rainbow trout. Seven trials were implemented; fish oil trial [FO; continuously fed by diet with fish oil (FO)], canola oil trial [CO; continuously fed by diet with canola oil (CO)], safflower oil trial (SFO; continuously fed by diet with safflower oil (SFO)], mixture oil trial (CSFO; continuously fed by diet containing both $\% 50 \mathrm{CO}$ and $\% 50$ $\mathrm{SFO})$, alternate cycled feeding trial [(FO/CO; fish fed by diet with $\mathrm{CO}$ for 2 weeks and $\mathrm{FO}$ for the following 2 weeks), (FO/SFO; fish fed by diet with SFO for 2 weeks and FO for following 2 weeks) and (FO/CSFO; fish fed by diet with CSFO for 2 weeks and FO for following 2 weeks)] for 12 weeks. Significantly higher SGR and the best FCR were detected in the CSFO and FO/CSFO feeding groups, respectively, compared to the FO group $(p>0.05)$. The lowest HSI and VSI were in the CO group ( $p>0.05)$. The highest crude protein, lipid and ash contents were determined in the FO/SFO group ( $>>0.05)$. Experimental diets containing vegetable oil (CO and SFO) and vegetable oil blend (CSFO) had significantly higher concentrations of n-6 fatty acids, predominantly in the form of linoleic (LA, 18:2n-6c) and oleic acid (OA, 18:1n-9c), while n-3 fatty acids were present in significantly higher concentrations in the FO group. The fatty acid composition of rainbow trout fillet reflected the dietary lipid source. Fillet of fish fed by the FO diet had highest eicosapentaenoic acid (EPA, 20:5n-3) and docosahexaenoic acid (DHA, 22:6n-3) amount. The n-3 PUFA accumulation (mainly EPA and DHA) was generally decreased in the fillets of the $\mathrm{CO}, \mathrm{SFO}, \mathrm{CSFO}$ groups compared to the FO group. The proportions of $\mathrm{n}-3$ PUFA in the fillets of the FO/ $\mathrm{CO}, \mathrm{FO} / \mathrm{SFO}$ and $\mathrm{FO} / \mathrm{CSFO}$ groups throughout the experimental period were significantly higher than in the $\mathrm{CO}, \mathrm{SFO}, \mathrm{CSFO}$ groups, confirming the major effect of the fish oil intake at the cycled feeding stages of the experiment. This study shows that cycled feeding regimes by diets including vegtable and FO may be used in rainbow trout culture.
\end{abstract}

\begin{tabular}{l} 
Article Information \\
\hline Received 06 February 2019 \\
Revised 15 May 2019 \\
Accepted 11 October 2019 \\
Available online 06 April 2020 \\
Authors' Contribution \\
SD and EK designed and supervised \\
the work. SD conducted the \\
experimental work and EK did the \\
biochemical analysis. SD and EK \\
wrote the article. \\
Key words \\
Rainbow trout, Vegetable oils, Cycled \\
feeding, Fatty acids, Grow
\end{tabular}

\section{INTRODUCTION}

$\mathrm{F}$ ish oil (FO) is the most important oil and used as energy source in aquafeed. Fish oil production in the world is based on fisheries and it is known that there is no improvement in the expected rate according to years and shows a stagnant slope (Caballero et al., 2002; Tacon, 2004; Montero et al., 2005). Compared with FO, vegetable oils are produced 100 times more in the world and are more balanced and even lower in terms of price, when used in feeds (Izquierdo et al., 2003; Turchini et al., 2009). The predicted shortage of FO has encouraged a global investigation into the use of alternative oil sources for aquaculture feed production (Francis et al., 2009). In recent years, the replacement of vegetable oil (VO) with FO

\footnotetext{
Corresponding author: sevalyaman@hotmail.com 0030-9923/2020/0004-1471 \$9.00/0

Copyright 2020 Zoological Society of Pakistan
}

in aquafeed has become an increasingly important issue due to a stagnant supply and the continuous increase in the demand of FO for aquaculture feeds. It has been reported that partial or complete replacement of VOs (soya, sunflower, linseed, canola, etc.) with FO in feeds yields successful results in terms of survival rate, growth performance and feed efficiency of fish. (Izquierdo et al., 2003, 2005).

Beside the possible positive effects on growth and feed effciency, the principal drawback of VO replacement in aquafeed is the resultant influence on fillet fatty acid composition (Francis et al., 2009). High levels of fish meal and oil, as main feed raw materials, are used in the cultivation of carnivorous fish. Almost all freshwater fish convert arachidonic acid from linoleic acid, EPA and DHA from $\alpha$-linolenic acid (Sargent et al., 2002). However, linoleic and $\alpha$-linolenic acids cannot be synthesized by the vertebrates including fish and are called essential fatty acids. Desaturation of these essential fatty acids 
(conversion to unsaturated form) and elongation can be converted to HUFA by alternative chains. $\Delta 6$ desaturation is responsible for the production of $18: 3 n-6$ from $18: 2 n-6$ and 18:4n-3 from 18:3n-3. Both productions are converted to $20: 4 n-6$ and $20: 5 n-3$ by $\Delta 5$ desaturation following fatty acid elongation (Sargent et al., 2002; Nakamura and Nara 2004). DHA is produced by further elongation, desaturation and short chain reactions (Sprecher et al., 1995). However, It was reported that using VO oil reduces the amount of n-3 polyunsaturated fatty acids (PUFA) in fish fillets, in particular DHA and EPA, which leads to an increase in n- 6 fatty acid compositions, also does not adversely affect the quality and taste of the meat (Rosenlund et al., 2001; Izquierdo et al., 2003; Kaushik, 2004). In this case, it was proposed to feed fish with "finishing" diets in a period of time before harvesting in order to restoration n-3 PUFA level in the fillets of fish fed by VO diets (Bell et al., 2004; Torstensen et al., 2004, 2005; Mourente et al., 2005; Izquierdo et al., 2005). "Finishing" diets appears to be a possible strategy to be evaluated in order to restoration the fatty acid composition of the fish fed by VO based diets and increase the used efficiency of FO in aquaculture. (Glencross et al., 2003; Robin et al., 2003; Bell et al., 2004; Jobling, 2004; Torstensen et al., 2005; Lane et al., 2006; Turchini et al., 2006, 2007; Trushenski and Boesenberg 2009).

Nevertheless, it should be noted that, even though finishing diets were successful in achieving the partial recovery of the main fatty acid composition of fish tissues, there is still a need for relatively large amount of fish oil during the several final months of production. Thus, alternative and innovative feeding strategies to address FO replacement, as well as other nutritional challenges, currently facing the aquaculture industry, are still need to be investigated (Eroldoğan et al., 2018). Consequently, the issue is the best addressed from as many perspectives as possible in order to provide an insight that may fill the knowledge blanks that limit and jeopardize the potential sustainability of fisheries and aquaculture sectors (Francis et al., 2009). As a simple and innovative approach, one of these could be via a better understanding and the possible usage of alternating feeding schedules (Eroldoğan et al., 2018).

The aim of this study was to evaluate the effects of alternate cycled feeding regime with diets including different oil sources on fatty acid profiles and growth performances of rainbow trout.

\section{MATERIALS AND METHODS}

Four experimental diets were formulated to be isonitrogenous (48\%) and isolipidic (18\%), but containing different sources of oil (Table I). These were; \%100 fish oil based diet (FO), \%100 canola oil based diet (CO), $\% 100$ safflower oil based diet (SFO) and \%50 canola oil+\%50 safflower oil mixture based diet (CSFO). Fish oil and feed ingredients were obtained from a local fish feed manufacturer (Sibal A.Ş. Black Sea Feed, Sinop, Turkey). Canola and safflower oils were purchased from a commercial company (Tokul Tarım A.Ş., İzmir/Turkey). Diets were prepared with a laboratory pelleting machine after adding 35- $40 \mathrm{~g}$ of distilled water into $1000 \mathrm{~g}$ mixture of ingredients and the pellets were dried at $40^{\circ} \mathrm{C}$ for $18 \mathrm{~h}$. Diets were crushed to the approximate size of $2 \mathrm{~mm}$ and kept at $-20^{\circ} \mathrm{C}$ until used.

Table I. Formulation (g/kg) and proximate composition (\%) of the experimental diets.

\begin{tabular}{lllll}
\hline & \multicolumn{5}{c}{ Experimental diets } \\
\cline { 2 - 5 } & FO (Control) & CO & SFO & CSFO \\
\hline Nutrient $\left(\mathrm{g} \mathrm{kg}^{-1}\right)$ & & & & \\
Fish meal & 320 & 320 & 320 & 320 \\
Extracted soybean meal & 250 & 250 & 250 & 250 \\
Wheat flour & 126 & 126 & 126 & 126 \\
Corn protein & 181 & 181 & 181 & 181 \\
Fish oil & 120 & 0 & 0 & 0 \\
Canola oil & 0 & 120 & 0 & 60 \\
Safflower oil & 0 & 0 & 120 & 60 \\
Vitamin premix $\left(^{*}\right)$ & 2 & 2 & 2 & 2 \\
Mineral premix $\left(^{*}\right)$ & 2 & 2 & 2 & 2 \\
Proximate composition & $(\%)$ & & & \\
Moisture & 4.82 & 4.83 & 5.28 & 5.13 \\
Protein & 48.52 & 48.55 & 48.97 & 48.53 \\
Lipid & 18.24 & 17.86 & 18.01 & 17.94 \\
Ash & 9.74 & 9.55 & 9.22 & 9.28 \\
NFE ${ }^{1}$ & 19.00 & 19.21 & 18.52 & 19.12 \\
Gross energy $\left(\mathrm{kJg}^{-1}\right)^{2}$ & 21.89 & 21.79 & 21.82 & 21.79 \\
\hline
\end{tabular}

${ }^{*}$ Vitamin-mineral premix (mg/kg premix): vitamin A, $210000 \mathrm{IU}$; Vitamin $\mathrm{D}_{3}, 35000 \mathrm{IU}$; vitamin $\mathrm{E}, 7000 \mathrm{mg}$; vitamin $\mathrm{K}_{3}, 322 \mathrm{mg}$; vitamin $\mathrm{B}_{1}, 588$ mg; vitamin $B_{2}, 252 \mathrm{mg}$; vitamin $B_{6}, 294 \mathrm{mg}$; vitamin $B_{12}, 826 \mathrm{mcg}$; niacin, $1400 \mathrm{mg}$; biotin, $7583 \mathrm{mcg}$; $182 \mathrm{mg}$ folic acid, pantothenic acid, $1722 \mathrm{mg}$; inositol, $17220 \mathrm{mg}$; vitamin C, $933.31 \mathrm{mg}$; Ca, 1414mg. ${ }^{1} \mathrm{NFE}$ $=100-(\%$ protein $+\%$ lipid $+\%$ ash $+\%$ moisture $) ;{ }^{2}$ Gross energy is calculated according to $23.6 \mathrm{~kJ} \mathrm{~g}^{-1}$ protein, $39.5 \mathrm{~kJ} \mathrm{~g}^{-1}$ lipid and $17 \mathrm{~kJ} \mathrm{~g}^{-1} \mathrm{NFE}$.

Rainbow trout used in this study were obtained from a commercial trout farm (Kuzey Su Ürünleri Inc., Samsun/ Turkey), transferred to Research and Application Center of Sinop University Fisheries Faculty (Sinop/Turkey) and kept for two weeks before the start of the experiment 


\begin{tabular}{|c|c|c|c|c|}
\hline $\begin{array}{l}\text { Fish oil trial (FO): fish were fed by the FO diet for } \\
\text { the all experiment during } 12 \text { weeks. }\end{array}$ & & & & \\
\hline $\begin{array}{l}\text { Canola oil trial (CO): fish were fed by the } \mathrm{CO} \text { diet for } \\
\text { the all experiment during } 12 \text { weeks. }\end{array}$ & 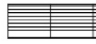 & 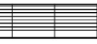 & 奉裳裳 & 整高 \\
\hline $\begin{array}{l}\text { Safflower oil trial (SFO): fish were fed by the SFO } \\
\text { diet for the all experiment during } 12 \text { weeks. }\end{array}$ & & & & \\
\hline $\begin{array}{l}\text { Canola-Safflower oil mixture trial (CSFO): fish were } \\
\text { fed by the CSFO diet for the all experiment during } 12 \\
\text { weeks. }\end{array}$ & \begin{tabular}{|l|l|}
- & \\
& \\
\end{tabular} & 1:1: & a: $\mid=0$ & $1:=$ \\
\hline $\begin{array}{l}\text { Alternate cycled trial (FO/CO): fish were fed } 2 \text { weeks } \\
\text { by the CO diet followed by } 2 \text { weeks with the FO diet. }\end{array}$ & 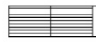 & 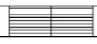 & 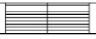 & \\
\hline $\begin{array}{l}\text { Alternate cycled trial (FO/SFO): fish were fed } 2 \\
\text { weeks by the SFO diet followed by } 2 \text { weeks with the } \\
\text { FO diet. }\end{array}$ & & & & \\
\hline $\begin{array}{l}\text { Alternate cycled trial (FO/CSFO): fish were fed } 2 \\
\text { weeks by the CSFO diet followed by } 2 \text { weeks with } \\
\text { the FO diet. }\end{array}$ & Ii: & & & \\
\hline
\end{tabular}

Fig. 1. The alternate cycled feeding that were applied during the study.

for acclimization. Fish were stocked in centrally drained three 40001 rectangle fiberglass tanks in a flow-through water system in an indoor facility during acclimation. After acclimatization, fish (mean weight of $63.20 \pm 0.12 \mathrm{~g}$ ) were fasted for a day, weighted and randomly distributed in triplicates to twenty-one fiberglass circular tanks (approximately, water volume of $300-\mathrm{L} ; 60 \mathrm{~cm}$ in high; $80 \mathrm{~cm}$ in diameter) at a density of 17 fish per tank. Water in flow was adjusted to $4 \mathrm{l} / \mathrm{min}$ and supplemental aeration was provided via airstone diffusers. The fish were individually weighted at the end and the beginning of the experiment with a $0.01 \mathrm{~g}$ sensitive electronic balance. The water quality parameters were monitored on weekly basis and the following parameters were recorded: average temperature of $15.94 \pm 0.07^{\circ} \mathrm{C}$, dissolved oxygen of $6.38 \pm 0.09 \mathrm{mg}^{-1}$ and $\mathrm{pH} 8.26$.

Fish were fed with one of the following seven feeding regimes: (1) Fish oil trial (FO): fish were fed by diet with FO during the all experimental period (12 weeks). (2) Canola oil trial (CO): fish were fed by diet with $\mathrm{CO}$ during the all experimental period (12 weeks). (3) Safflower oil trial (SFO): fish were fed by diet with SFO during the all experimental period (12 weeks). (4) Mixture oil trial (CSFO): fish were fed by diet with CSFO (as in Table I) during the all experimental period (12 weeks). (5) Alternate cycled trial $(\mathrm{FO} / \mathrm{CO})$ : fish were fed by diet with $\mathrm{CO}$ for 2 weeks and FO for the following 2 weeks (12 weeks). (6) Alternate cycled trial (FO/SFO): fish were fed by diet with SFO for 2 weeks and FO for the following 2 weeks (12 weeks). (7) Alternate cycled trial (FO/CSFO): fish were fed by diet with CSFO for 2 weeks and FO for the following for 2 weeks (12 weeks) (Fig. 1).

Fish in all groups were fed by hand twice a day (at 09:00 am and 15:30 pm) until apparent satiety under a natural light regime (10 h dark:14 h light) throughout the experiment. At the beginning of the experiment, twenty fish from stock pool for initial sample were sacrificed, weighed and the whole fish were homogenized. After completion of the experiment, five fish from each experiment tank were sampled. Initial and final samples were stored at $-80^{\circ} \mathrm{C}$ until analysed.

The analyses of proximate composition on feed ingredients, diets and fish were performed according to the standard methods of AOAC (1995). dry matter after drying in an oven at $105^{\circ} \mathrm{C}$ until constant weight, crude protein ( $\mathrm{N} \times 6.25)$ by Kjeldhal method after acid digestion, ash content by incineration in a muffle furnace at $550^{\circ} \mathrm{C}$ for 6 $\mathrm{h}$, crude lipid after extraction with petroleum ether by the Soxhlet method. Gross energy of the diets was estimated assuming $23.6 \mathrm{~kJ} / \mathrm{g}$ protein, $39.5 \mathrm{~kJ} / \mathrm{g}$ lipid and $17 \mathrm{~kJ} / \mathrm{g}$ nitrogen free extracts.

Total lipid was determined by modified Bligh and Dyer Method (Hanson and Olley, 1963). $0.25 \mathrm{~g}$ of extracted oil from fish fillets and diets was thawed by adding $4 \mathrm{ml}$ of heptane and $0.4 \mathrm{ml}$ of $2 \mathrm{~N} \mathrm{KOH}$ was added. This mixture was stirred in vortex for 2 minutes, then centrifuged at $5000 \mathrm{rpm}$ for 5 minutes. After centrifugation, $1.5-2 \mathrm{ml}$ of the heptane phase was collected and transferred to glass tubes for GC/MS analysis. The injection of samples into the device was performed with the autosampler AI 1310.

Samples were analyzed by Thermo Scientific ISQ LT model GC/MS gas chromatography by spectrometer. For this analysis, with $0.25 \mu \mathrm{m}$ film thickness was used a Trace Gold TG-WaxMS capillary column (Thermo Scientific code: 26088-1540) in $0.25 \mu \mathrm{m}$ inner diameter and $60 \mu \mathrm{m}$ length. The injection block temperature was adjusted to $240^{\circ} \mathrm{C}$ and the column temperature program to be increased from $100^{\circ} \mathrm{C}$ to $240^{\circ} \mathrm{C}$. Helium gas $(1 \mathrm{ml} / \mathrm{min})$ was used as a carrier gas and 1:20 split ratio was applied. The MS unit (ISQ LT) was used in electron ionization mode. Fatty acids were defined by comparing the standard FAME mixture of 37 components [Chem-Lab Fame mix (37C) standard 
solution; Art. Nr. CL40. 13093; Lot Nr. 221.561.102.100] with respect to their arrival time.

Amino acid analyzes were performed on Agilent Technologies / 6460 Triple Quad LC-MS / MS Liquid Chromatography Mass / Mass Spectrometer; 5 different standard, internal standard, mobile phases, reagents, chromotographic separation and mass detection parameters with the modified sample preparation including acidic hydrolysis process is made using the Jasem LC-MS / MS amino acid analysis kit (Pt Berca Niaga Medica/Indonesia) (Bilgin et al., 2018).

Table II. Amino acid composition of the experimental diets (g/100g).

\begin{tabular}{|c|c|c|c|c|}
\hline & \multicolumn{4}{|c|}{ Diet groups } \\
\hline & FO(Control) & $\mathrm{CO}$ & SFO & CSFO \\
\hline \multicolumn{5}{|c|}{ Essential amino acids (EAA, g/100g) } \\
\hline Arginine & $3.31 \pm 0.03^{\mathrm{a}}$ & $3.58 \pm 0.08^{\mathrm{a}}$ & $3.21 \pm 0.02^{\mathrm{a}}$ & $3.53 \pm 0.05^{\mathrm{a}}$ \\
\hline Histidine & $1.98 \pm 0.00^{\mathrm{a}}$ & $2.12 \pm 0.04^{\mathrm{a}}$ & $1.90 \pm 0.06^{\mathrm{a}}$ & $2.04 \pm 0.01^{\mathrm{a}}$ \\
\hline Isoleucine & $2.15 \pm 0.01^{\mathrm{b}}$ & $2.46 \pm 0.02^{\mathrm{a}}$ & $2.07 \pm 0.12^{\mathrm{b}}$ & $2.50 \pm 0.01^{\mathrm{a}}$ \\
\hline Leucine & $5.19 \pm 0.11^{\mathrm{b}}$ & $5.56 \pm 0.14^{\mathrm{a}}$ & $4.85 \pm 0.06^{\mathrm{b}}$ & $5.47 \pm 0.06^{\mathrm{a}}$ \\
\hline Lysine & $3.97 \pm 0.07^{\mathrm{b}}$ & $4.44 \pm 0.02^{\mathrm{a}}$ & $4.20 \pm 0.02^{\mathrm{ab}}$ & $4.50 \pm 0.08^{\mathrm{a}}$ \\
\hline Methionine & $1.07 \pm 0.08^{\mathrm{a}}$ & $1.26 \pm 0.04^{\mathrm{a}}$ & $1.09 \pm 0.05^{\mathrm{a}}$ & $1.17 \pm 0.01^{\mathrm{a}}$ \\
\hline Cystine & $0.53 \pm 0.03^{\mathrm{a}}$ & $0.46 \pm 0.02^{\mathrm{b}}$ & $0.49 \pm 0.01^{\mathrm{ab}}$ & $0.53 \pm 0.02^{\mathrm{a}}$ \\
\hline Phenylalanine & $2.86 \pm 0.09^{\mathrm{ab}}$ & $3.03 \pm 0.04^{\mathrm{a}}$ & $2.73 \pm 0.00^{\mathrm{b}}$ & $2.98 \pm 0.05^{\mathrm{a}}$ \\
\hline Valine & $2.86 \pm 0.10^{\mathrm{b}}$ & $3.08 \pm 0.04^{\mathrm{a}}$ & $2.82 \pm 0.02^{\mathrm{b}}$ & $3.13 \pm 0.06^{\mathrm{a}}$ \\
\hline Threonine & $2.67 \pm 0.03^{b}$ & $2.67 \pm 0.07^{\mathrm{b}}$ & $2.54 \pm 0.15^{\mathrm{b}}$ & $2.90 \pm 0.11^{\mathrm{a}}$ \\
\hline \multicolumn{5}{|c|}{ Non-essential amino acids (NEAA, g/100g) } \\
\hline Alanine & $3.65 \pm 0.02^{\mathrm{a}}$ & $3.79 \pm 0.05^{\mathrm{a}}$ & $3.50 \pm 0.02^{\mathrm{a}}$ & $3.84 \pm 0.02^{\mathrm{a}}$ \\
\hline Aspartic acid & $5.25 \pm 0.14^{\mathrm{a}}$ & $5.73 \pm 0.19^{\mathrm{a}}$ & $5.44 \pm 0.22^{\mathrm{a}}$ & $5.22 \pm 0.02^{\mathrm{a}}$ \\
\hline Glutamic acid & $9.46 \pm 0.26^{b}$ & $9.72 \pm 0.46^{\mathrm{b}}$ & $9.49 \pm 0.18^{\mathrm{b}}$ & $10.43 \pm 0.32^{\mathrm{a}}$ \\
\hline Glysine & $3.00 \pm 0.16^{\mathrm{b}}$ & $3.00 \pm 0.04^{\mathrm{b}}$ & $2.93 \pm 0.05^{\mathrm{b}}$ & $3.45 \pm 0.01^{\mathrm{a}}$ \\
\hline Serine & $2.91 \pm 0.05^{\mathrm{a}}$ & $2.95 \pm 0.00^{\mathrm{a}}$ & $2.74 \pm 0.00^{\mathrm{b}}$ & $3.01 \pm 0.01^{\mathrm{a}}$ \\
\hline Tyrosine & $1.54 \pm 0.02^{\mathrm{a}}$ & $1.67 \pm 0.03^{\mathrm{a}}$ & $1.62 \pm 0.02^{\mathrm{a}}$ & $1.60 \pm 0.02^{\mathrm{a}}$ \\
\hline Taurine & $0.23 \pm 0.01^{\mathrm{a}}$ & $0.22 \pm 0.00^{\mathrm{a}}$ & $0.20 \pm 0.01^{\mathrm{a}}$ & $0.21 \pm 0.00^{\mathrm{a}}$ \\
\hline Proline & $3.62 \pm 0.04^{\mathrm{b}}$ & $3.81 \pm 0.00^{\mathrm{a}}$ & $3.50 \pm 0.02^{\mathrm{b}}$ & $3.95 \pm 0.05^{\mathrm{a}}$ \\
\hline Ornithine & $0.15 \pm 0.05^{\mathrm{a}}$ & $0.16 \pm 0.00^{\mathrm{a}}$ & $0.16 \pm 0.00^{\mathrm{a}}$ & $0.16 \pm 0.00^{\mathrm{a}}$ \\
\hline
\end{tabular}

FO, Fish oil; CO, Canola oil; SFO, Safflower oil; CSFO, CanolaSafflower oil mixture. Values are means of three determinations \pm SE. Means with different superscript letter in a row are significantly different $(\mathrm{p}>0.05)$

\section{Statistical analysis}

Anderson-Darling and Levene's tests were used for homogeneity of variances and equality of variance of groups, respectively. The effects of dietary oils on muscle fatty acid compositions and the significance of differences in growth between control and treated groups were analyzed using one-way analysis of variance (ANOVA), followed by Tukey's method for multiple comparisons. Arcsine square root transformations of percentage data were conducted for homogeneity of variances before statistical analysis. Differences were considered significant when $\mathrm{p}<0.05$. Analyses were performed using Minitab 17 software for Windows.

Table III. Fatty acid composition of the experimental diets (\% total fatty acids).

\begin{tabular}{|c|c|c|c|c|}
\hline & \multicolumn{4}{|c|}{ Diet Groups } \\
\hline & FO (Control) & $\mathrm{CO}$ & SFO & CSFO \\
\hline $\mathrm{C} 14: 0$ & $5.49 \pm 0.03^{\mathrm{a}}$ & $1.63 \pm 0.02^{\mathrm{c}}$ & $1.85 \pm 0.04^{b}$ & $1.57 \pm 0.06^{\mathrm{d}}$ \\
\hline C15:0 & $1.35 \pm 0.01^{\mathrm{a}}$ & $0.17 \pm 0.00^{\mathrm{b}}$ & $0.18 \pm 0.00^{\mathrm{b}}$ & $0.16 \pm 0.01^{\mathrm{c}}$ \\
\hline C16:0 & $12.14 \pm 0.01^{\mathrm{b}}$ & $10.25 \pm 0.06^{\mathrm{c}}$ & $12.91 \pm 0.11^{\mathrm{a}}$ & $10.27 \pm 0.09^{\mathrm{c}}$ \\
\hline $\mathrm{C} 17: 0$ & $1.53 \pm 0.06^{\mathrm{a}}$ & $0.17 \pm 0.00^{\mathrm{b}}$ & $0.20 \pm 0.01^{\mathrm{b}}$ & $0.17 \pm 0.01^{\mathrm{b}}$ \\
\hline C18:0 & $7.02 \pm 0.15^{\mathrm{b}}$ & $4.65 \pm 0.03^{\mathrm{d}}$ & $7.67 \pm 0.15^{\mathrm{a}}$ & $5.68 \pm 0.15^{\mathrm{c}}$ \\
\hline C20:0 & $0.52 \pm 0.02^{\mathrm{a}}$ & $0.31 \pm 0.01^{\mathrm{b}}$ & $0.09 \pm 0.00^{c}$ & $0.11 \pm 0.00^{\mathrm{c}}$ \\
\hline $\mathrm{C} 21: 0$ & $0.09 \pm 0.01^{\mathrm{a}}$ & $0.10 \pm 0.01^{\mathrm{a}}$ & $0.01 \pm 0.00^{\mathrm{c}}$ & $0.05 \pm 0.01^{\mathrm{b}}$ \\
\hline $\mathrm{C} 22: 0$ & $1.90 \pm 0.06^{\mathrm{a}}$ & $1.32 \pm 0.06^{\mathrm{b}}$ & $1.02 \pm 0.03^{\mathrm{c}}$ & $1.03 \pm 0.05^{\mathrm{c}}$ \\
\hline$\Sigma$ SFA & $30.03 \pm 0.34^{\mathrm{a}}$ & $18.58 \pm 0.16^{\mathrm{c}}$ & $23.93 \pm 0.33^{b}$ & $19.04 \pm 0.36^{\mathrm{c}}$ \\
\hline C14:1 & $0.45 \pm 0.01^{\mathrm{a}}$ & $0.04 \pm 0.00^{\mathrm{b}}$ & $0.04 \pm 0.00^{\mathrm{b}}$ & $0.04 \pm 0.00^{\mathrm{b}}$ \\
\hline C15:1 & $0.21 \pm 0.00^{\mathrm{a}}$ & $0.02 \pm 0.00^{\mathrm{b}}$ & $0.02 \pm 0.00^{\mathrm{b}}$ & $0.02 \pm 0.00^{\mathrm{b}}$ \\
\hline C16:1 & $5.86 \pm 0.12^{\mathrm{a}}$ & $2.34 \pm 0.01^{\mathrm{b}}$ & $2.35 \pm 0.07^{\mathrm{b}}$ & $2.10 \pm 0.09^{\mathrm{c}}$ \\
\hline C17:1 & $0.66 \pm 0.04^{\mathrm{a}}$ & $0.15 \pm 0.00^{\mathrm{b}}$ & $0.10 \pm 0.01^{\mathrm{c}}$ & $0.12 \pm 0.01^{\mathrm{bc}}$ \\
\hline C18:1 n-9c & $13.21 \pm 0.05^{\mathrm{d}}$ & $31.92 \pm 0.38^{\mathrm{a}}$ & $22.22 \pm 0.62^{\mathrm{c}}$ & $28.43 \pm 0.25^{\mathrm{b}}$ \\
\hline C18:1 n-9t & $2.40 \pm 0.26^{\mathrm{c}}$ & $5.37 \pm 0.45^{\mathrm{a}}$ & $2.35 \pm 0.44^{\mathrm{c}}$ & $4.06 \pm 0.00^{\mathrm{b}}$ \\
\hline C20:1 & $3.17 \pm 0.03^{\mathrm{a}}$ & $3.10 \pm 0.03^{b}$ & $1.34 \pm 0.04^{\mathrm{d}}$ & $2.34 \pm 0.00^{\mathrm{c}}$ \\
\hline C20:1 n-9 & $2.33 \pm 0.09^{\mathrm{a}}$ & $0.77 \pm 0.02^{b}$ & $0.96 \pm 0.03^{b}$ & $0.88 \pm 0.11^{\mathrm{b}}$ \\
\hline $\mathrm{C} 24: 1$ & $1.51 \pm 0.05^{\mathrm{a}}$ & $0.77 \pm 0.02^{\mathrm{bc}}$ & $0.82 \pm 0.02^{\mathrm{b}}$ & $0.72 \pm 0.04^{\mathrm{c}}$ \\
\hline$\Sigma$ MUFA & $29.79 \pm 0.19^{c}$ & $44.46 \pm 0.12^{\mathrm{a}}$ & $30.2 \pm 0.03^{c}$ & $38.71 \pm 0.50^{\mathrm{b}}$ \\
\hline C18:2 n-6c & $8.47 \pm 0.03^{\mathrm{d}}$ & $16.96 \pm 0.03^{\mathrm{c}}$ & $30.71 \pm 0.45^{\mathrm{a}}$ & $23.55 \pm 1.25^{\mathrm{b}}$ \\
\hline $\mathrm{C} 18: 2 \mathrm{n}-6 \mathrm{t}$ & $0.62 \pm 0.01^{\mathrm{a}}$ & $0.14 \pm 0.01^{\mathrm{b}}$ & $0.10 \pm 0.00^{c}$ & $0.11 \pm 0.00^{c}$ \\
\hline C18:3 n-3 & $1.65 \pm 0.04^{\mathrm{a}}$ & $1.65 \pm 0.02^{\mathrm{a}}$ & $1.55 \pm 0.04^{b}$ & $1.55 \pm 0.05^{\mathrm{b}}$ \\
\hline C18:3 n-6 & $3.24 \pm 0.01^{\mathrm{c}}$ & $7.48 \pm 0.07^{\mathrm{a}}$ & $2.01 \pm 0.04^{\mathrm{d}}$ & $6.03 \pm 0.06^{\mathrm{b}}$ \\
\hline $\mathrm{C} 20: 2$ & $0.83 \pm 0.05^{\mathrm{a}}$ & $0.37 \pm 0.01^{\mathrm{b}}$ & $0.28 \pm 0.01^{\mathrm{c}}$ & $0.32 \pm 0.02^{\mathrm{bc}}$ \\
\hline$C 20: 3 n-6$ & $0.60 \pm 0.01$ & $\mathrm{Nd}$ & $\mathrm{Nd}$ & $\mathrm{Nd}$ \\
\hline C20:4 n-6 & $1.90 \pm 0.06^{\mathrm{a}}$ & $0.54 \pm 0.02^{\mathrm{b}}$ & $0.62 \pm 0.03^{b}$ & $0.54 \pm 0.03^{b}$ \\
\hline$C 20: 5 n-3$ & $7.42 \pm 0.15^{\mathrm{a}}$ & $2.63 \pm 1.57^{b}$ & $4.60 \pm 0.02^{b}$ & $4.45 \pm 0.14^{b}$ \\
\hline $\mathrm{C} 22: 2$ & $0.19 \pm 0.02^{\mathrm{a}}$ & $0.13 \pm 0.00^{\mathrm{b}}$ & $0.11 \pm 0.01^{\mathrm{c}}$ & $0.14 \pm 0.01^{b}$ \\
\hline $\mathrm{C} 22: 6 \mathrm{n}-3$ & $14.14 \pm 0.17^{\mathrm{a}}$ & $5.17 \pm 0.00^{\mathrm{c}}$ & $5.55 \pm 0.03^{b}$ & $5.17 \pm 0.08^{c}$ \\
\hline$\Sigma$ PUFA & $39.04 \pm 0.13^{c}$ & $35.04 \pm 1.56^{\mathrm{d}}$ & $45.54 \pm 0.28^{\mathrm{a}}$ & $41.86 \pm 0.87^{b}$ \\
\hline$\Sigma$ n-3 PUFA & $23.21 \pm 0.28^{\mathrm{a}}$ & $9.45 \pm 1.59^{c}$ & $11.7 \pm 0.09^{b}$ & $11.17 \pm 0.27^{\mathrm{bc}}$ \\
\hline$\Sigma \mathrm{n}-6$ PUFA & $14.82 \pm 0.11^{\mathrm{d}}$ & $25.10 \pm 0.03^{\mathrm{c}}$ & $33.44 \pm 0.39^{\mathrm{a}}$ & $30.23 \pm 1.17^{b}$ \\
\hline$n-3 / n-6$ & $1.57 \pm 0.03^{\mathrm{a}}$ & $0.38 \pm 0.06^{\mathrm{b}}$ & $0.35 \pm 0.01^{b}$ & $0.37 \pm 0.02^{\mathrm{b}}$ \\
\hline DHA/EPA & $1.91 \pm 0.01^{\mathrm{b}}$ & $3.06 \pm 1.82^{\mathrm{a}}$ & $1.21 \pm 0.00^{\mathrm{c}}$ & $1.16 \pm 0.02^{\mathrm{c}}$ \\
\hline
\end{tabular}

nd, not detected; FO, Fish oil; CO, Canola oil; SFO, Safflower oil; CSFO, Canola-Safflower oil mixture; SFA, Saturated fatty acids; MUFA, Monounsaturated fatty acids; PUFA, Polyunsaturated fatty acids; DHA, Docosahexaenoic acid; EPA, Eicosapentaenoic acid. Values are means of three determinations \pm SE. 
Table IV. Growth performance, feed efficiency and other body parameters of rainbow trout fed the seven experimental diets for 12 weeks.

\begin{tabular}{|c|c|c|c|c|c|c|c|}
\hline & \multicolumn{4}{|l|}{ Diets Groups } & \multicolumn{3}{|c|}{ Cycled Feeding Groups } \\
\hline & FO (Control) & $\mathrm{CO}$ & SFO & CSFO & $\mathrm{FO} / \mathrm{CO}$ & FO/SFO & FO/CSFO \\
\hline IBW (g) & $62.89 \pm 0.27^{\mathrm{a}}$ & $62.93 \pm 0.17^{\mathrm{a}}$ & $63.42 \pm 0.17^{\mathrm{a}}$ & $62.91 \pm 0.06^{\mathrm{a}}$ & $63.21 \pm 0.49^{\mathrm{a}}$ & $63.74 \pm 0.18^{\mathrm{a}}$ & $63.32 \pm 0.27^{\mathrm{a}}$ \\
\hline FBW (g) & $167.97 \pm 6.91^{\mathrm{e}}$ & $180.74 \pm 28.85^{\mathrm{d}}$ & $188.77 \pm 4.06^{\mathrm{c}}$ & $208.64 \pm 4.47^{\mathrm{a}}$ & $202.29 \pm 6.72^{\mathrm{b}}$ & $201.39 \pm 6.43^{\mathrm{b}}$ & $206.85 \pm 2.59^{\mathrm{a}}$ \\
\hline WG $(g)^{1}$ & $105.08 \pm 6.97^{\mathrm{e}}$ & $117.81 \pm 28.86^{\mathrm{d}}$ & $125.34 \pm 4.17^{\mathrm{c}}$ & $145.73 \pm 4.49^{\mathrm{a}}$ & $139.08 \pm 6.44^{b}$ & $137.66 \pm 6.39^{b}$ & $143.53 \pm 2.36^{\mathrm{a}}$ \\
\hline $\operatorname{SGR}(\%)^{2}$ & $1.33 \pm 0.05^{\mathrm{e}}$ & $1.39 \pm 0.24^{\mathrm{d}}$ & $1.47 \pm 0.03^{\mathrm{c}}$ & $1.62 \pm 0.03^{\mathrm{a}}$ & $1.57 \pm 0.04^{\mathrm{b}}$ & $1.55 \pm 0.04^{b}$ & $1.60 \pm 0.01^{\mathrm{a}}$ \\
\hline $\mathrm{FCR}^{3}$ & $1.16 \pm 0.01^{\mathrm{b}}$ & $1.35 \pm 0.29^{\mathrm{a}}$ & $1.16 \pm 0.01^{\mathrm{b}}$ & $1.11 \pm 0.04^{\mathrm{c}}$ & $1.09 \pm 0.01^{\mathrm{d}}$ & $1.09 \pm 0.01^{\mathrm{d}}$ & $1.02 \pm 0.01^{\mathrm{e}}$ \\
\hline $\mathrm{FI}^{4}$ & $121.29 \pm 1.11^{\mathrm{e}}$ & $136.45 \pm 2.62^{\mathrm{d}}$ & $145.09 \pm 0.53^{\mathrm{c}}$ & $161.41 \pm 1.50^{\mathrm{a}}$ & $150.78 \pm 1.10^{\mathrm{b}}$ & $151.14 \pm 0.82^{\mathrm{b}}$ & $146.50 \pm 0.80^{\mathrm{c}}$ \\
\hline HSI $(\%)^{5}$ & $0.99 \pm 0.05^{\mathrm{a}}$ & $0.85 \pm 0.14^{b}$ & $0.99 \pm 0.05^{\mathrm{a}}$ & $0.98 \pm 0.01^{\mathrm{a}}$ & $0.90 \pm 0.02^{\mathrm{a}}$ & $0.93 \pm 0.02^{\mathrm{a}}$ & $0.99 \pm 0.01^{\mathrm{a}}$ \\
\hline VSI $(\%)^{6}$ & $9.19 \pm 0.09^{\mathrm{a}}$ & $8.68 \pm 1.55^{\mathrm{b}}$ & $9.97 \pm 0.31^{\mathrm{a}}$ & $10.09 \pm 0.15^{\mathrm{a}}$ & $9.38 \pm 0.14^{\mathrm{a}}$ & $9.06 \pm 0.36^{\mathrm{a}}$ & $9.24 \pm 0.25^{\mathrm{a}}$ \\
\hline
\end{tabular}

Data are reported as mean \pm standard errors of three replicates (3). Means with different superscript letter in a row are significantly different ( $\mathrm{p}>0.05$ ). IBW, Initial body weight; FBW, Final body weight; FI, Feed intake. ${ }^{1}$ Weight gain (WG, g) $=$ Final body weight - initial body weight; ${ }^{2}$ Specific growth rate $(\mathrm{SGR})=[(\ln$ final wet weight - ln initial wet weight $) /$ days $] \times 100 ;{ }^{3}$ Feed conversion rate $(\mathrm{FCR})=$ total feed intake/weight gain; ${ }^{4}$ Feed Intake $(\mathrm{FI}, \mathrm{g} / \mathrm{fish})=$ Total feed intake $(\mathrm{g}) /$ number of fish; ${ }^{5}$ Hepatosomatic index $(\mathrm{HSI})=($ liver weight $/$ body weight $) \times 100$; ${ }^{6}$ Viserosomatic index $(\mathrm{VSI})=(\mathrm{viscera}$ weight $/$ body weight) x 100 .

\section{RESULTS}

The essential amino acid profiles of the experimental diets meet the requirements for rainbow trout (NRC, 1993). All the essential amino acids for growth were found in the diets. The best amino acid composition was found in CSFO group (Table II). In general, however, the amino acid composition of all groups was sufficient to meet the amino acid requirements of rainbow trout.

Total saturated fatty acids (SFA) ranged from $18.58 \%$ to $30.03 \%$ and was the highest in the FO diet, mainly made up of myristic acid (MA, 14:0) high level. Although the FO diet had the highest SFA, the SFO diet had the highest level of PA. Monounsaturated fatty acids (MUFA) were higher in the $\mathrm{CO}$ and $\mathrm{CSFO}$ diets than in FO and SFO diets which was ranged from $31.93 \%$ in $\mathrm{CO}$ to $28.43 \%$ in CSFO diets, and oleic acid (OA, 18:1n-9c) formed the main component. Linoleic acid (LA, 18:2n-6c) was the highest in SFO diet $(30.71 \%)$. The FO diet had the lowest LA $(8.47 \%)$ content (Table III). The FO and CO diets had the highest level of $\alpha$-linolenic acid ( $\alpha$-ALA, 18:3n-3) (1.65\%), whereas the SFO and CSFO diets had the lowest (1.55\%). Comparing with the FO diet, experimental diets were poor in arachidonic acid (ARA, 20:4n-6), eicosapentaenoic acid (EPA, 20:5n-3), docosahexaenoic acid (DHA, 22:6n3) n-3 PUFA and n-3/n-6 levels ( $p>0.05)$. Notably, EPA and DHA levels were high with values of $7.42 \pm 0.15$ and $14.14 \pm 0.17$, respectively, in the FO diet.

Significant differences were detected in both the growth performance and feeding efficiencies between FO and dietary treatments (Table IV). The weight gain (WG) and specific growth rate (SGR) in the fish fed by
VO supplemented and cycled feeding diets were higher than FO group $(\mathrm{p}<0.05)$. Among all experimental groups, SGR and WG varied between 1.33 and $1.62 \%$ day $^{-1}$ and 105.08 to $145.73 \mathrm{~g}$ respectively. Significantly lower FCR was detected in $\mathrm{CO}$ group $(\mathrm{p}<0.05)$. The highest and lowest feed intake (FI) were obtained in the CSFO group and FO group, respectively $(\mathrm{p}<0.05)$. The highest growth performance was detected in the CSFO group and all cycled feeding groups. The lowest hepatosomatic index (HSI) and vicerosomatic index (VSI) values were determined in the CO group. HSI and VSI were significantly differed between other groups and $\mathrm{CO}$ group and ranged from $0.85-0.99$ and $8.68-10.22 \%$, respectively $(\mathrm{p}<0.05)$.

Table V. Chemical composition of fillet of rainbow trout fed the seven experimental diets for 12 weeks (\% wet weight).

\begin{tabular}{lllll}
\hline & Moisture (\%) & Protein (\%) & Lipid (\%) & Ash (\%) \\
\hline Initial & $77.62 \pm 0.10^{\mathrm{a}}$ & $21.82 \pm 0.36^{\mathrm{c}}$ & $2.29 \pm 0.33^{\mathrm{d}}$ & $1.85 \pm 0.03^{\mathrm{c}}$ \\
FO (Control) & $74.19 \pm 0.11^{\mathrm{b}}$ & $23 . .62 \pm 0.32^{\mathrm{b}}$ & $4.94 \pm 0.01^{\mathrm{c}}$ & $2.38 \pm 0.24^{\mathrm{a}}$ \\
CO & $72.77 \pm 1.13^{\mathrm{d}}$ & $23.41 \pm 0.40^{\mathrm{b}}$ & $5.65 \pm 0.44^{\mathrm{b}}$ & $2.39 \pm 0.23^{\mathrm{a}}$ \\
$\mathrm{SFO}$ & $73.62 \pm 0.15^{\mathrm{b}}$ & $24.76 \pm 0.11^{\mathrm{a}}$ & $5.51 \pm 0.56^{\mathrm{b}}$ & $1.94 \pm 0.08^{\mathrm{b}}$ \\
CSFO & $72.82 \pm 0.18^{\mathrm{b}}$ & $24.58 \pm 0.55^{\mathrm{a}}$ & $5.52 \pm 0.51^{\mathrm{b}}$ & $2.14 \pm 0.25^{\mathrm{a}}$ \\
FO/CO & $73.36 \pm 0.49^{\mathrm{c}}$ & $23.70 \pm 0.33^{\mathrm{b}}$ & $5.19 \pm 0.58^{\mathrm{b}}$ & $2.18 \pm 0.24^{\mathrm{a}}$ \\
FO/SFO & $73.41 \pm 0.43^{\mathrm{c}}$ & $25.01 \pm 0.59^{\mathrm{a}}$ & $6.07 \pm 0.13^{\mathrm{a}}$ & $2.43 \pm 0.23^{\mathrm{a}}$ \\
FO/CSFO & $73.05 \pm 0.30^{\mathrm{d}}$ & $22.92 \pm 0.52^{\mathrm{b}}$ & $5.11 \pm 0.40^{\mathrm{b}}$ & $2.32 \pm 0.06^{\mathrm{a}}$ \\
\hline Da
\end{tabular}

Data are mean \pm SE. Means with different superscript letter in a row are significantly different $(\mathrm{p}>0.05)$. 
Table VI. Fatty acid composition of fillet of rainbow trout fed the seven experimental diets (\% of total fatty acids) for 12 weeks.

\begin{tabular}{|c|c|c|c|c|c|c|c|c|}
\hline & \multicolumn{3}{|c|}{ Diets Groups } & \multirow[b]{2}{*}{ SFO } & \multicolumn{4}{|c|}{ Cycled Feeding Groups } \\
\hline & Initial & FO(Control) & CO & & CSFO & FO/CO & $\mathrm{CO} / \mathrm{SFO}$ & FO/CSFO \\
\hline C14:0 & $2.54 \pm 0.02^{\mathrm{d}}$ & $3.88 \pm 0.02^{\mathrm{a}}$ & $1.88 \pm 0.03^{\mathrm{e}}$ & $1.68 \pm 0.00^{\mathrm{g}}$ & $1.77 \pm 0.01^{\mathrm{f}}$ & $3.15 \pm 0.01^{\mathrm{b}}$ & $3.04 \pm 0.01^{\mathrm{c}}$ & $3.11 \pm 0.00^{\mathrm{b}}$ \\
\hline $\mathrm{C} 15: 0$ & $0.46 \pm 0.01^{\mathrm{d}}$ & $1.14 \pm 0.01^{\mathrm{a}}$ & $0.30 \pm 0.01^{\mathrm{e}}$ & $0.26 \pm 0.00^{\mathrm{f}}$ & $0.28 \pm 0.01^{\mathrm{f}}$ & $0.84 \pm 0.01^{\mathrm{b}}$ & $0.80 \pm 0.01^{\mathrm{c}}$ & $0.84 \pm 0.01^{\mathrm{b}}$ \\
\hline $\mathrm{C} 16: 0$ & $11.13 \pm 0.02^{\mathrm{d}}$ & $13.18 \pm 0.04^{\mathrm{a}}$ & $11.10 \pm 0.21^{\mathrm{de}}$ & $10.52 \pm 0.02^{\mathrm{f}}$ & $10.88 \pm 0.01^{\mathrm{e}}$ & $12.39 \pm 0.03^{\mathrm{bc}}$ & $12.22 \pm 0.03^{\mathrm{c}}$ & $12.48 \pm 0.03^{b}$ \\
\hline $\mathrm{C} 17: 0$ & $0.55 \pm 0.01^{\mathrm{e}}$ & $1.13 \pm 0.00^{\mathrm{a}}$ & $0.26 \pm 0.01^{\mathrm{f}}$ & $0.22 \pm 0.01^{\mathrm{g}}$ & $0.18 \pm 0.00^{\mathrm{h}}$ & $0.79 \pm 0.01^{\mathrm{c}}$ & $0.77 \pm 0.01^{\mathrm{d}}$ & $0.81 \pm 0.00^{\mathrm{b}}$ \\
\hline C18:0 & $7.28 \pm 0.09^{\mathrm{d}}$ & $7.19 \pm 0.00^{\mathrm{d}}$ & $7.26 \pm 0.09^{\mathrm{d}}$ & $7.46 \pm 0.01^{\mathrm{c}}$ & $7.27 \pm 0.04^{\mathrm{d}}$ & $7.64 \pm 0.02^{b}$ & $7.73 \pm 0.06^{\mathrm{ab}}$ & $7.85 \pm 0.04^{b}$ \\
\hline C20:0 & $1.00 \pm 0.02^{\mathrm{f}}$ & $0.51 \pm 0.00^{\mathrm{g}}$ & $1.35 \pm 0.02^{\mathrm{d}}$ & $2.50 \pm 0.00^{\mathrm{a}}$ & $1.82 \pm 0.02^{\mathrm{b}}$ & $1.03 \pm 0.04^{\mathrm{ef}}$ & $1.50 \pm 0.01^{\mathrm{c}}$ & $1.06 \pm 0.01^{\mathrm{e}}$ \\
\hline $\mathrm{C} 21: 0$ & $2.55 \pm 0.03^{b}$ & $0.10 \pm 0.01^{\mathrm{e}}$ & $0.24 \pm 0.00^{\mathrm{c}}$ & $4.32 \pm 0.01^{\mathrm{a}}$ & $0.28 \pm 0.02^{\mathrm{c}}$ & $0.13 \pm 0.01^{\mathrm{de}}$ & $0.15 \pm 0.01^{\mathrm{d}}$ & $0.16 \pm 0.01^{\mathrm{d}}$ \\
\hline $\mathrm{C} 22: 0$ & $1.99 \pm 0.06^{\mathrm{ef}}$ & $2.02 \pm 0.01^{\mathrm{e}}$ & $1.93 \pm 0.02^{\mathrm{f}}$ & $3.30 \pm 0.00^{\mathrm{a}}$ & $2.99 \pm 0.01^{\mathrm{b}}$ & $1.85 \pm 0.01^{\mathrm{g}}$ & $2.36 \pm 0.02^{\mathrm{c}}$ & $2.18 \pm 0.01^{\mathrm{d}}$ \\
\hline$\Sigma \mathrm{SFA}$ & $27.47 \pm 0.12^{\mathrm{d}}$ & $29.13 \pm 0.00^{\mathrm{b}}$ & $24.31 \pm 0.37^{\mathrm{f}}$ & $30.24 \pm 0.01^{\mathrm{a}}$ & $25.46 \pm 0.09^{\mathrm{e}}$ & $27.80 \pm 0.09^{\mathrm{d}}$ & $28.56 \pm 0.09^{c}$ & $28.46 \pm 0.04^{\mathrm{c}}$ \\
\hline $\mathrm{C} 14: 1$ & $0.15 \pm 0.00^{\mathrm{d}}$ & $0.36 \pm 0.00^{\mathrm{a}}$ & $0.08 \pm 0.00^{\mathrm{e}}$ & $0.06 \pm 0.00^{\mathrm{g}}$ & $0.07 \pm 0.00^{\mathrm{f}}$ & $0.26 \pm 0.00^{\mathrm{b}}$ & $0.25 \pm 0.01^{\mathrm{c}}$ & $0.26 \pm 0.00^{\mathrm{b}}$ \\
\hline $\mathrm{C} 15: 1$ & $0.09 \pm 0.00^{\mathrm{cd}}$ & $0.30 \pm 0.07^{\mathrm{a}}$ & $0.10 \pm 0.05^{\mathrm{cd}}$ & $0.05 \pm 0.01^{\mathrm{d}}$ & $0.04 \pm 0.00^{\mathrm{d}}$ & $0.18 \pm 0.03^{\mathrm{bc}}$ & $0.25 \pm 0.01^{\mathrm{ab}}$ & $0.24 \pm 0.04^{\mathrm{ab}}$ \\
\hline C16:1 & $3.96 \pm 0.02^{\mathrm{e}}$ & $5.37 \pm 0.02^{\mathrm{a}}$ & $3.33 \pm 0.06^{\mathrm{f}}$ & $2.61 \pm 0.01^{\mathrm{h}}$ & $3.04 \pm 0.01^{\mathrm{g}}$ & $4.61 \pm 0.01^{\mathrm{b}}$ & $4.35 \pm 0.01^{\mathrm{d}}$ & $4.49 \pm \pm 0.00^{\mathrm{c}}$ \\
\hline $\mathrm{C} 17: 1$ & $0.39 \pm 0.01^{\mathrm{d}}$ & $0.75 \pm 0.01^{\mathrm{a}}$ & $0.27 \pm 0.00^{\mathrm{e}}$ & $0.19 \pm 0.01^{g}$ & $0.22 \pm 0.00^{\mathrm{f}}$ & $0.59 \pm 0.01^{\mathrm{b}}$ & $0.55 \pm 0.01^{\mathrm{c}}$ & $0.59 \pm 0.01^{\mathrm{b}}$ \\
\hline C18:1 n-9c & $14.99 \pm 0.10^{\mathrm{d}}$ & $12.66 \pm 0.00^{\mathrm{e}}$ & $24.89 \pm 1.05^{\mathrm{a}}$ & $15.52 \pm 0.20^{\mathrm{d}}$ & $20.01 \pm 0.02^{\mathrm{b}}$ & $16.71 \pm 0.04^{\mathrm{c}}$ & $12.94 \pm 0.04^{\mathrm{e}}$ & $14.60 \pm 0.04^{\mathrm{d}}$ \\
\hline C18:1n-9t & $2.80 \pm 0.13^{\mathrm{abc}}$ & $2.63 \pm 0.02^{\mathrm{bc}}$ & $2.46 \pm 0.04^{\mathrm{bc}}$ & $2.39 \pm 0.41^{\mathrm{c}}$ & $2.42 \pm 0.02^{\mathrm{bc}}$ & $2.60 \pm 0.03^{\mathrm{bc}}$ & $2.85 \pm 0.08^{\mathrm{ab}}$ & $3.17 \pm 0.04^{\mathrm{a}}$ \\
\hline $\mathrm{C} 20: 1$ & $3.50 \pm 0.05^{\mathrm{b}}$ & $2.75 \pm 0.00^{\mathrm{e}}$ & $3.66 \pm 0.05^{\mathrm{a}}$ & $2.27 \pm 0.00^{\mathrm{g}}$ & $3.14 \pm 0.01^{\mathrm{c}}$ & $3.17 \pm 0.02^{\mathrm{c}}$ & $2.51 \pm 0.00^{\mathrm{f}}$ & $2.85 \pm 0.01^{\mathrm{d}}$ \\
\hline C20:1 n-9 & $2.85 \pm 0.02^{\mathrm{a}}$ & $2.58 \pm 0.01^{\mathrm{b}}$ & $1.57 \pm 0.03^{\mathrm{e}}$ & $1.14 \pm 0.01^{\mathrm{g}}$ & $1.25 \pm 0.01^{\mathrm{f}}$ & $2.17 \pm 0.01^{\mathrm{c}}$ & $2.04 \pm 0.02^{\mathrm{d}}$ & $2.03 \pm 0.00^{\mathrm{d}}$ \\
\hline $\mathrm{C} 24: 1$ & $1.04 \pm 0.02^{\mathrm{e}}$ & $1.37 \pm 0.01^{\mathrm{c}}$ & $0.89 \pm 0.00^{\mathrm{f}}$ & $1.95 \pm 0.01^{\mathrm{a}}$ & $1.40 \pm 0.01^{\mathrm{c}}$ & $1.19 \pm 0.00^{\mathrm{d}}$ & $1.54 \pm 0.0^{\mathrm{b}}$ & $1.39 \pm 0.01^{\mathrm{c}}$ \\
\hline$\Sigma$ MUFA & $29.76 \pm 0.29^{c}$ & $28.74 \pm 0.09^{c}$ & $37.23 \pm 0.83^{\mathrm{a}}$ & $26.16 \pm 0.62^{\mathrm{e}}$ & $31.58 \pm 0.02^{\mathrm{b}}$ & $31.47 \pm 0.01^{\mathrm{b}}$ & $27.26 \pm 0.07^{d}$ & $29.59 \pm 0.03^{\mathrm{c}}$ \\
\hline C18:2 n-6c & $12.37 \pm 0.11^{\mathrm{d}}$ & $9.68 \pm 0.07^{\mathrm{e}}$ & $12.72 \pm 0.13^{\mathrm{cd}}$ & $17.88 \pm 0.64^{\mathrm{a}}$ & $16.27 \pm 0.11^{\mathrm{b}}$ & $10.91 \pm 0.02^{\mathrm{e}}$ & $14.10 \pm 0.02^{\mathrm{c}}$ & $13.39 \pm 1.24^{\mathrm{cd}}$ \\
\hline C18:2 n-6t & $0.50 \pm 0.01^{\mathrm{b}}$ & $0.11 \pm 0.00^{\mathrm{g}}$ & $0.28 \pm 0.01^{\mathrm{d}}$ & $0.20 \pm 0.00^{\mathrm{f}}$ & $0.24 \pm 0.00^{\mathrm{e}}$ & $0.51 \pm 0.00^{\mathrm{a}}$ & $0.48 \pm 0.01^{\mathrm{c}}$ & $0.50 \pm 0.00^{\mathrm{b}}$ \\
\hline $\mathrm{C} 18: 3 \mathrm{n}-3$ & $0.79 \pm 0.01^{\mathrm{d}}$ & $1.05 \pm 0.01^{\mathrm{a}}$ & $1.06 \pm 0.02^{\mathrm{a}}$ & $0.81 \pm 0.01^{\mathrm{d}}$ & $0.92 \pm 0.00^{c}$ & $1.09 \pm 0.00^{\mathrm{a}}$ & $0.99 \pm 0.01^{\mathrm{b}}$ & $1.07 \pm 0.01^{\mathrm{a}}$ \\
\hline C18:3 n-6 & $4.13 \pm 0.02^{\mathrm{ab}}$ & $3.24 \pm 0.02^{\mathrm{cd}}$ & $4.75 \pm 0.08^{\mathrm{a}}$ & $2.02 \pm 0.00^{\mathrm{e}}$ & $3.53 \pm 0.00^{\mathrm{bc}}$ & $3.19 \pm 0.67^{\mathrm{cd}}$ & $2.66 \pm 0.01^{\mathrm{de}}$ & $3.21 \pm 0.01^{\mathrm{cd}}$ \\
\hline $\mathrm{C} 20: 2$ & $2.55 \pm 0.03^{\mathrm{f}}$ & $2.13 \pm 0.00^{\mathrm{g}}$ & $2.83 \pm 0.03^{\mathrm{e}}$ & $4.32 \pm 0.01^{\mathrm{a}}$ & $3.78 \pm 0.01^{\mathrm{b}}$ & $2.53 \pm 0.01^{\mathrm{f}}$ & $3.25 \pm 0.00^{\mathrm{c}}$ & $2.91 \pm 0.01^{\mathrm{d}}$ \\
\hline$C 20: 3 n-6$ & $1.71 \pm 0.04^{\mathrm{f}}$ & $1.13 \pm 0.00^{\mathrm{g}}$ & $2.20 \pm 0.03^{\mathrm{d}}$ & $3.65 \pm 0.01^{\mathrm{a}}$ & $2.97 \pm 0.00^{\mathrm{b}}$ & $1.70 \pm 0.01^{\mathrm{f}}$ & $2.45 \pm 0.01^{\mathrm{c}}$ & $2.11 \pm 0.00^{\mathrm{e}}$ \\
\hline C20:4 n-6 & $1.99 \pm 0.06^{\mathrm{bc}}$ & $2.02 \pm 0.01^{\mathrm{bc}}$ & $1.93 \pm 0.02^{\mathrm{c}}$ & $3.30 \pm 0.00^{\mathrm{a}}$ & $2.99 \pm 0.01^{\mathrm{a}}$ & $1.46 \pm 0.39^{d}$ & $2.36 \pm 0.02^{\mathrm{b}}$ & $2.18 \pm 0.01^{\mathrm{bc}}$ \\
\hline$C 20: 5 n-3$ & $4.51 \pm 0.02^{\mathrm{b}}$ & $5.68 \pm 0.02^{\mathrm{a}}$ & $2.90 \pm 0.04^{\mathrm{d}}$ & $2.23 \pm 0.00^{\mathrm{f}}$ & $2.50 \pm 0.00^{\mathrm{e}}$ & $4.51 \pm 0.01^{\mathrm{b}}$ & $4.33 \pm 0.03^{\mathrm{c}}$ & $4.32 \pm 0.01^{\mathrm{c}}$ \\
\hline $\mathrm{C} 22: 2$ & $0.09 \pm 0.01^{\mathrm{d}}$ & $0.18 \pm 0.00^{\mathrm{ab}}$ & $0.16 \pm 0.01^{\mathrm{bc}}$ & $0.16 \pm 0.01^{\mathrm{bc}}$ & $0.15 \pm 0.00^{c}$ & $0.17 \pm 0.00^{\mathrm{abc}}$ & $0.20 \pm 0.03^{\mathrm{a}}$ & $0.18 \pm 0.00^{\mathrm{ab}}$ \\
\hline$C 22: 6 n-3$ & $13.42 \pm 0.18^{\mathrm{b}}$ & $15.87 \pm 0.02^{\mathrm{a}}$ & $9.18 \pm 0.12^{\mathrm{e}}$ & $8.61 \pm 0.02^{\mathrm{f}}$ & $9.20 \pm 0.03^{\mathrm{e}}$ & $12.80 \pm 0.05^{\mathrm{c}}$ & $12.55 \pm 0.06^{\mathrm{d}}$ & $12.47 \pm 0.03^{\mathrm{d}}$ \\
\hline$\Sigma$ PUFA & $42.03 \pm 0.15^{\mathrm{ab}}$ & $41.07 \pm 0.10^{\mathrm{b}}$ & $37.97 \pm 0.46^{\mathrm{c}}$ & $43.16 \pm 0.65^{\mathrm{a}}$ & $42.53 \pm 0.08^{\mathrm{ab}}$ & $38.86 \pm 1.15^{\mathrm{c}}$ & $43.34 \pm 0.05^{\mathrm{a}}$ & $42.32 \pm 1.21^{\mathrm{ab}}$ \\
\hline$\Sigma$ n-3 PUFA & $18.71 \pm 0.21^{\mathrm{b}}$ & $22.59 \pm 0.04^{\mathrm{a}}$ & $13.13 \pm 0.17^{\mathrm{e}}$ & $11.65 \pm 0.02^{\mathrm{g}}$ & $12.62 \pm 0.04^{\mathrm{f}}$ & $18.40 \pm 0.07^{\mathrm{c}}$ & $17.87 \pm 0.08^{\mathrm{d}}$ & $17.86 \pm 0.04^{\mathrm{d}}$ \\
\hline$\Sigma$ n-6 PUFA & $20.68 \pm 0.02^{\mathrm{b}}$ & $16.18 \pm 0.06^{\mathrm{d}}$ & $21.86 \pm 0.26^{\mathrm{b}}$ & $27.04 \pm 0.63^{\mathrm{a}}$ & $25.99 \pm 0.10^{\mathrm{a}}$ & $19.16 \pm 0.33^{c}$ & $22.03 \pm 0.01^{\mathrm{b}}$ & $21.39 \pm 1.25^{\mathrm{b}}$ \\
\hline$n-3 / n-6$ & $0.90 \pm 0.01^{\mathrm{b}}$ & $1.40 \pm 0.00^{\mathrm{a}}$ & $0.60 \pm 0.00^{\mathrm{d}}$ & $0.43 \pm 0.01^{\mathrm{e}}$ & $0.49 \pm 0.00^{\mathrm{e}}$ & $0.96 \pm 0.02^{\mathrm{b}}$ & $0.81 \pm .0 .00^{\mathrm{c}}$ & $0.84 \pm 0.05^{\mathrm{c}}$ \\
\hline DHA/EPA & $2.98 \pm 0.02^{\mathrm{d}}$ & $2.80 \pm 0.00^{\mathrm{g}}$ & $3.17 \pm 0.00^{\mathrm{c}}$ & $3.86 \pm 0.01^{\mathrm{a}}$ & $3.69 \pm 0.00^{\mathrm{b}}$ & $2.84 \pm 0.01^{\mathrm{f}}$ & $2.90 \pm 0.01^{\mathrm{e}}$ & $2.89 \pm 0.00^{\mathrm{e}}$ \\
\hline
\end{tabular}

Data are reported as mean \pm standard errors of three replicate (3). Means with different superscript letter in a row are significantly different ( $p>0.05)$. SFA, Saturated fatty acids; MUFA, Monounsaturated fatty acids; PUFA, Polyunsaturated fatty acids; DHA, Docosahexaenoic acid; EPA, Eicosapentaenoic acid.

Fish fillet proximate compositions were significantly influenced by dietary groups (Table V, $\mathrm{p}<0.05$ ). Moisture, crude protein, crude lipid and crude ash were different in all experimental groups compared to the initial samples. In addition, the moisture content in the FO group and crude protein, lipid and ash content in $\mathrm{FO} / \mathrm{SFO}$ group were 
higher than in the other groups $(\mathrm{p}<0.05)$. The highest crude protein, lipid and ash were determined in $\mathrm{FO} / \mathrm{SFO}$ group.

The fatty acid composition of fillets was influenced by the cycled feeding regimes (Table VI). The higher SFA level was detected in SFO group $(\mathrm{p}<0.05)$, whereas CO group had the lowest content. While $\mathrm{CO}$ group had the highest fillet MUFA content, which was predominantly made up of OA, the lowest MUFA was determined in SFO group. The highest LA increase was observed in SFO group having the highest LA value $(p<0.05)$. EPA, DHA, n-3 PUFA and n3/n6 rates were significantly high $(\mathrm{p}<0.05)$ in FO group, comparison to the $\mathrm{CO}, \mathrm{SFO}$ and CSFO groups. EPA, DHA, n-3 PUFA and n3/n6 rates were almost close to FO group in the cycled feeding groups. PUFA was higher in the FO, SFO, CSFO, FO/SFO and $\mathrm{FO} / \mathrm{CSFO}$ groups than $\mathrm{CO}$ and $\mathrm{FO} / \mathrm{CO}$ groups. Also, high PUFAs detected in the SFO, CSFO, FO/SFO and FO/ CSFO groups were due to n- 6 fatty acids.

\section{DISCUSSION}

In recent years, an important part of the researches related to nutrition of carnivorous fish species is related to effect of vegetable oils used as an alternative to fish oil, which one of the basic raw materials of fish diets, on fish growth performance and fatty acid profiles It is already known that oils used as energy sources in fish diets have an effect on growth, survival and body composition (Nandeesha et al., 2002; Ali et al., 2005; Francis et al., 2009; Eroldoğan et al., 2013; Masiha et al., 2015; Eroldoğan et al., 2018). In the present study it was aimed to evaluate whether it was possible to increase growth performance and the efficiency of n-3 PUFA deposition in the fish body via the implementation of different cycled feeding, where FO and VO based diets were regularly alternated on a biweekly base.

The best FCR was in FO/CSFO, FO/CO, FO/SFO and CSFO groups, respectively. FCR is one of the most widely used marker in determining fish growth performance. Feeding habits, feed intake and digestive enzymes activity are also other effective factors in growth. Şener (1996) reported that vegetable oils involved in trout diets increased digestibility. Consequently, it was observed that fish in the CSFO and cycled feeding groups were larger than the other groups. The FO, CO and SFO groups showed the lowest growth performance although all experimental conditions were the same. The results of the study indicate that feeding fish with the mixed VO based and cycled feeding diets had positive effect on growth performance. Numerous studies reported that the replacement of fish oil with vegetable oils in aquafeed had minimal harmful impacts on growth performance on fish (Tocher et al., 2002; Regost et al.,
2003; Izquierdo et al., 2005; Mourente and Bell, 2006; Guler and Y1ldız, 2011; Turchini et al., 2011; Eroldoğan et al., 2012; Masiha et al., 2013; Y1lmaz et al., 2016). Francis et al. (2009) reported that although there was no difference in palatability between the groups, the fish exposed to alternating cycles of 2 weeks of FO and 2 weeks of canola oil diets were larger than the fish subjected to any other treatments in Murray cod (Maccullochella peelii peelii). It has been reported that canola oil has no adverse effects on growth, feed utilization, biometry and fatty acid composition in rainbow trout (Turchini et al., 2013). Benitez-Dorta et al. (2013) reported that total replacement of fish oil by vegetable oils does not induce any adverse changes on growth performance in Senegalese sole (Solea senegalensis).

In the present study, among cycled feeding groups, fish in FO/CSFO group grew significantly faster than fish in $\mathrm{FO} / \mathrm{CO}$ and $\mathrm{FO} / \mathrm{SFO}$ groups during the final finishing period. The growth difference among the groups might be due to the difference in the FCR of the fish in the FO/ CSFO group that was higher (less efficient) than the other treatments (Glencross et al., 2003; Turchini et al., 2007; Turchini and Francis, 2009; Y1lmaz et al., 2016). Lysine is one of the most important essential amino acids that positively affects the growth performance by increasing the fillet weight in fish (Furuya et al., 2010). In the present study, looking the amino acid content of the feed, the best essential amino acids and hence the highest lysine amount were in the CSFO feed. In previous researches, it was observed that fish fed with high energy feeds showed faster growth and the energy required for fish growth were supplied from amino acids and fats (Korkut et al., 2007). Growth potential differs depending on fish species and some other factors. Furthermore, the degree to which growth potential is realized is highly dependent on feed intake and on how well the feed has been adjusted to the nutritional needs of the fish. (Eriegha and Ekokotu, 2017). In the present study, the highest feed intake was in the CSFO group. Accordingly, the best growth was also in this group. Shahrooz et al. (2018) indicated that replacement of fish oil with $\mathrm{CO}$, SFO, LSO (linseed oil) and vegetable oil mixtures (CSLO) in rainbow trout has not any adverse effects on growth performance and foregut histo architecture. In addition, the growth performances of the CO and CSLO groups were better than the other groups and the number of goblet cells decreased in these groups; however, the thickness and height of the intestinal folds and the thickness of the tunica mucosa and tunica lower mucosa were reported to be increased compared to the FO group. Examining the growth performance and histological findings, no adverse effects were reported due to the using canola and mixed vegetable oils compared to 
the FO group.

The moisture, lipid and protein contents of fillets were significantly influenced by the dietary lipid source. Fish fed with FO/SFO diet had the highest crude lipid and protein contents in fillets compared to fish fed the other experimental diets, followed by $\mathrm{CO}$ group for lipid and SFO group for protein. Similar results for lipid contents were reported in some studies that fish were fed different vegetable oils (Güler and Y1ldız, 2011; Turchini et al., 2011; Köse and Yıldız, 2013; Yılmaz et al., 2016). These studies showed that high dietary level of $18: 2 n-6$ or $18: 1 n-9$ fatty acids accumulated particularly in the liver and body tissue. Francis et al. (2009) reported that no significant differences were detected between the whole bodies of juvenile Murray cod subjected to the different feeding schedules in terms of moisture, protein, lipid and ash content.

Fatty acid composition of fish tissues was reported to be affected by fatty acids mobilization, catabolism and saturation access or chain elongation of fatty acid and also shaped according to fatty acids taken from feeds (Sargent et al., 2002; Bell and Waagbø, 2008; Turchini et al., 2009). Numerous studies, in a wide range of fish species, showed that flesh fatty acid composition is closely correlated to dietary fatty acid composition and feeding the fish by high levels of vegetable oils will strongly influence the preferential deposition and retention of "unwanted" fatty acids such as 18:2n-6 and 18:3n-3 in flesh lipids (Y1lmaz et al., 2016). Especially, in fish fed by vegetable oils, while the $\mathrm{n}-6$ series fatty acids increased, there was a significant decrease in the amount of n-3 high unsaturated fatty acids such as EPA and DHA (Sargent et al. 2002). In the present study, n-6 series fatty acids increased in the groups fed with vegetable oil supplemented feeds, while the EPA and DHA ratios decreased. Thus, there have been many attempts to overcome this problem in aquaculture practices, such as implementation of "cleaner" feeds (finishing diets as reported in Fountoulaki et al., 2009; Bell et al., 2003; Ng et al., 2004; Turchini et al., 2007; Codabaccus et al., 2013; Ng et al., 2013; Y1ldiz et al., 2018) or alternate feeding regime including different lipid sources (Francis et al., 2009; Brown et al., 2010; Mumoğullarında, 2012; Dedeler, 2013). In the present study, an alternate cycled feeding regime was applied, as a result of which there was a significant decrease in n-6 series fatty acids and EPA and DHA rates reached high levels. Recycling of n-3 series fatty acids was not achieved by $100 \%$ since 4 -week of cycled feeding regime was applied in the study. However, $76-79 \%$ recycle was determined based on the FO group.

Previous studies showed that although dietary fatty acids associate with fatty acids deposited in fish tissue, some specific fatty acids (i.e. linoleic acid, linolenic acid, EPA and DHA) were preferentially retained (Y1lmaz et al.,
2016). In the present study, it was clearly showed that the fatty acid composition of the fillets of rainbow trout fed with seven feeding regimes was reflected the dietary fatty acid composition. Cycled feeding groups had significantly higher EPA and DHA contents in the fillets in comparison to fish fed the CO, SFO and CSFO groups. Similar results were reported in some studies that fish were fed by different vegetable oils (Brown et al., 2010; Francis et al., 2009; Mumoğullarında, 2012; Dedeler, 2013; Yılmaz et al., 2016; Eroldoğan et al., 2018). It was reported that the cycled feeding mechanism has a positive effect on the recycling of nutrients to the composition of fatty acids in alternative feed (Sargent et al., 2002; Turchini et al., 2007). Francis et al. (2009) determined that n-3 PUFAs of Murray cod fed by fish oil for 2 weeks and vegetable oil diets for 2 weeks were at the same level with FO group. Brown et al., (2010) reported that the rainbow trout fed with fish oil in the morning meal and vegetable oil diets in the evening meal were similar fatty acid profiles in the fish fed by fat diet.

Cyclic feeding studies are generally carried out to recover $n-3$ PUFAs that occur in fish fed with vegetable oil supplementation. For this purpose, n-3 PUFA level in fish fillet is tried to be ensured the level of fish fed by diet including fish oil by giving the diet including fish and vegetable oils cyclically at certain time intervals until the harvest period. In a number of research on cycled feeding studies, n-3 PUFAs in fillet of cycled fed fish by diets including fish/vegetable oils not exactly complete recovery as in fish fed by diet including fish oil. However, it was stated that a better level of n-3 PUFA is obtained compared to feeding fish with only vegetable oil supplemented diet (Francis et al., 2009; Brown et al., 2010). In the present study, n-3 PUFA level of cycled feeding groups did not reach the level of n-3 PUFA determined in the group fed with the fish oil (FO), but it was much better than the n-3 PUFA level of the groups fed with only vegetable oil supplemented diet (CO, SFO, CSFO).

\section{CONCLUSION}

This study revealed the possibility of reducing the use of fish oil in aquafeeds by using alternative feeding regimes. Also, the present findings in our study showed that when vegetable oil and FO based diets were routinely alternated, rainbow trout were able to increase their efficiency of $n-3$ LC-PUFA deposition in the flesh.

\section{ACKNOWLEDGEMENT}

This study was supported by the Support Project (SÜF-1901-18-47) of Sinop University and the fatty and amino acids analyzes of this study were carried out by the 
Scientific and Technological Research and Application Center (SUBİTAM). This study was conducted at the Research and Application Center of the Aquaculture and Fisheries Faculty of Sinop University, Turkey. We extend our gratefulness to Osman Parlak, the owner of the Kuzey Fish Farm, for providing the fish.

\section{Statement of conflict of interset}

The declare there is no conflict of interset.

\section{REFERENCES}

Ali, Z., Hossain, A. and Mazid, A., 2005. Effect of mixed feeding schedules with varying dietary protein levels on the growth of sutchi catfish, Pangasius hypophthalmus (Sauvage) with silver carp, Hypophthalmichthys molitrix (Valenciennes) in ponds. Aquacul. Res., 36: 627-634. https://doi. org/10.1111/j.1365-2109.2005.01262.x

AOAC, 1995. Animal feed. (ed. W. Horwitz). Official methods of analysis of the association of analytical chemists. $13^{\text {th }}$ Edition 7: 125. USA.

Benítez-Dorta, V., Caballero, M.J., Izquierdo, M., Manchado, M., Infante, C., Zamorano, M.J. and Montero, D., 2013. Total substitution of fish oil by vegetable oils in Senegalese sole (Solea senegalensis) diets: Effects on fish performance, biochemical composition, and expression of some glucocorticoid receptor-related genes. Fish Physiol. Biochem., 39: 335-349. https://doi.org/10.1007/ s10695-012-9703-4

Bell, J.G., Tocher, D.R., Henderson, R.J., Dick, J.R. and Cramptom, V.O., 2003. Altered fatty acid compositions in Atlantic salmon (Salmo salar) fed diets containing linseed and rapeseed oils can be partially restored by a subsequent fish oil finishing diet. J. Nutri., 133: 2793-2801. https://doi. org/10.1093/jn/133.9.2793

Bell, J.G., Henderson, R.J., Tocher, D.R. and Sargent, J.R., 2004. Replacement of dietary fish oil with increasing levels of linseed oil: Modification of flesh fatty acid compositions in Atlantic salmon (Salmo salar) using a fish oil finishing diet. Lipids, 39: 223232. https://doi.org/10.1007/s11745-004-1223-5

Bell, G.J. and Waagbø, R., 2008. Aquaculture in the ecosystem. Safe and nutritious aquaculture produce: Benefits and risks of alternative sustainable aquafeeds. Chapter 6. SpringerLink, pp. 185-225. https://doi.org/10.1007/978-1-4020-6810-2_6

Bilgin, Ö., Çarlı, U., Erdoğan, S., Maviş, M.E., Gürsu, G.G. and Y1lmaz, M., 2018. Determination of amino acid contents of anchovy, Engraulis encrasicolus, using LC-MS/MS. Turkish J. agric. Nat. Sci., 5: 465-470. https://doi.org/10.30910/turkjans.471272

Brown, T.D., Francis, D.S. and Turchini, G.M., 2010. Can dietary lipid source circadian alternation improve omega-3 deposition in rainbow trout. Aquaculture, 300:148-155. https://doi.org/10.1016/j. aquaculture.2009.12.020

Caballero, M.J., Obach, A., Roselund, G., Montero, D., Gisvold, M. and Izquierdo, M.S., 2002. Impact of different dietary lipid sources on growth, lipid digestibility, tissue fatty acid composition and histology of rainbow trout, Oncorhynchus mykiss. Aquaculture, 214: 253-271. https://doi.org/10.1016/ S0044-8486(01)00852-3

Codabaccus, M.B., Ng, W-K., Nichols, P.D. and Carter, C.G., 2013. Restoration of EPA and DHA in rainbow trout (Oncorhynchus mykiss) using a finishing fish oil diet at two different water temperatures. $F d$. Chem., 141: 236-244. https://doi.org/10.1016/j. foodchem.2013.02.032

Dedeler, H., 2013. The effect of diets including fish oil and vegetable oil sources and their use in cycles growth and fatty acid composition of juvenile European sea bass (Dicentrarchus labrax). An MS thesis submitted to the Department of Aquaculture. Cukurova Universty, Adana, Turkey. pp. 68.

Eriegha, O.J. and Ekokotu, P.A., 2017. Factors affecting feed intake in cultured fish species: A review. Anim. Res. Int., 14: 2697-2709.

Eroldoğan, O.T., Turchini, G.M., Yılmaz, A.H., Taşbozan, O., Engin, K., Ölçülü, A. and Özşahinoğlu, I., Mumoğullarında, P., 2012. Potential of cottonseed oil as fish oil replacer in European sea bass (Dicentrarchus labrax) feed formulation. Turkish $J$. Fish. Aquat. Sci., 12: 787-797.

Eroldoğan, O.T., Yılmaz, A.H. and Turchini, G.M., 2013. Fatty acid metabolism in European sea bass (Dicentrarchus labrax): Effects of n-6 PUFA and MUFA in fish oil replaced diets. Fish Physiol. Biochem., 39: 941-955. https://doi.org/10.1007/ s10695-012-9753-7

Eroldoğan, O.T., Elsabagh, M., Emre, Y., Turchini, G.M., Yılmaz, A.H., Eraslan, D., Emre, N. and Evliyaoğlu, E., 2018. Circadian feeding schedules in gilthead sea bream (Sparus aurata) and European sea bass (Dicentrarchus labrax): A comparative approach towards improving dietary fish oil utilization and n-3 LC-PUFA metabolism. Aquaculture, 495: 806-814. https://doi.org/10.1016/j.aquaculture.2018.06.070

Fountoulaki, E., Vasilaki, A., Hurtado, R., Grigorakis, K., Karacostas, I., Nengas, I., Rigos, G., Kotzamanis, Y., Venou, B. and Alexis, M.N., 2009. Fish oil 
substitution by vegetable oils in commercial diets for gilthead sea bream (Sparus aurata L.); effects on growth performance, flesh quality and fillet fatty acid profile: Recovery of fatty acid profiles by a fish oil finishing diet under fluctuating water temperatures. Aquaculture, 289: 317-326. https:// doi.org/10.1016/j.aquaculture.2009.01.023

Francis, D.S., Turchini., G.M., Jones, P.L., Smith, B.K., Stephen, G.R. and De Silva, S.S., 2009. Effects of alternate phases of fish oil and vegetable oil-based diets in Murray cod. Aquacul. Res., 40: 1123-1134. https://doi.org/10.1111/j.1365-2109.2009.02208.x

Furuya, W.M. and Furuya, V.R.B., 2010. Nutritional innovations on amino acids supplementation in Nile tilapia diets. Rev. Brasil. Zootec., 39: 88-94. https:// doi.org/10.1590/S1516-35982010001300010

Glencross, B.D., Hawkins, W.E. and Curnow, J.G., 2003. Restoration of the fatty acid composition of red seabream ( Pagrus auratus ) using a fish oil finishing diet after grow-out on plant oil based diets. Aquacul. Nutri., 9: 409-418. https://doi.org/10.1046/j.13652095.2003.00272.x

Güler, M. and Yıldız, M., 2011. Eff ects of dietary fi sh oil replacement by cottonseed oil on growth performance and fatty acid composition of rainbow trout (Oncorhynchus mykiss). Turk. J. Vet. Anim. Sci., 35: 157-167.

Hanson S.W.F. and Olley, J., 1963. Application of the Bligh and Dyer method of lipid extraction to tissue homogenates. Biochem. J., 89: 101-102.

Izquierdo, M.S., Obach, A., Arantzamendi, L., Montero, D., Rabaina, L. and Rosenlund, G., 2003. Dietary lipid sources for seabream and seabass: growth performance, tissue composition and flesh quality. Aquac. Nutr., 9: 397-407. https://doi.org/10.1046/ j.1365-2095.2003.00270.x

Izquierdo, M.S., Montero, D., Robaina, L., Caballero, M.J., Rosenlund, G. and Ginés, R., 2005. Alterations in fillet fatty acid profile and flesh quality in gilthead seabream (Sparus aurata) fed vegetable oils for a long term period. Recovery of fatty acid profiles by fish oil feeding. Aquaculture, 250: 431-444. https:// doi.org/10.1016/j.aquaculture.2004.12.001

Jobling, M., 2004. Finishing feeds for carnivorous fish and the fatty acid dilution model. Aquacul. Res., 35: 706-709. https://doi.org/10.1111/j.13652109.2004.01067.x

Kaushik, S.J., 2004. Fish oil replacement in aquafeeds. Aqua Feeds Formul. Beyond, 1: 3-6.

Korkut, A.Y., Kop, A., Demirtaş, N. and Cihaner, A., 2007. Determination methods of growth performance in fish feding. E.U. J. Fish. Aquat. Sci., 24: 201-205.
Köse, I. and Yıldız, M., 2013. Effect of diets containing sesame oil on growth and fatty acid composition of rainbow trout (Oncorhynchus mykiss). J. Appl. Ichthyol., 29: 1318-1324. https://doi.org/10.1111/ jai.12184

Lane, R.L., Trushenski, J.T. and Kohler., C.C., 2006. Modification of fillet composition and evidence of differential fatty acid turnover in sunshine bass Morone chtysops $\mathrm{x}$ M. saxatilis following change in dietary lipid source. Lipids, 41: 1029-1038. https:// doi.org/10.1007/s11745-006-5053-2

Masiha, A., Soofiani, N.M., Ebrahimi, E., Kadivar, M. and Karimi, M.R., 2013. Effect of dietary flaxseed oil level on the growth performance and fatty acid composition of fingerlings of rainbow trout, Oncorhynchus mykiss. Springer Plus, 2: 1-7. https:// doi.org/10.1186/2193-1801-2-1

Masiha A., Ebrahimi E., Soofiani N.M. and Kadivar M., 2015. Effect of dietary canola oil level on the growth performance and fatty acid composition of fingerlings of rainbow trout (Oncorhynchus mykiss). Iranian J. Fish. Sci., 14: 336-349.

Montero, D., Robaina, L., Caballero, M.J., Gines, R. and Izquierdo, M.S., 2005. Growth, feed utilization and flesh quality of European sea bass (Dicentrarchus labrax) fed diets containing vegetable oils: a time course study on the effect of a re-feeding period with 100\% fish oil diet. Aquaculture, 248: 121-134. https://doi.org/10.1016/j.aquaculture.2005.03.003

Mourente, G., Dick, J.R., Bell, J.G. and Tocher, D.R., 2005. Effect of partial substitution of dietary fish oil by vegetable oils ondesaturation and $\beta$ - oxidation of [1-14C] 20:5n-3 (EPA) inhepatocytes and enterocytes of European sea bass (Dicentrarchus labrax L.). Aquaculture, 248: 173-186. https://doi. org/10.1016/j.aquaculture.2005.04.023

Mourente, G. and Bell, J.G., 2006. Partial replacement of dietary fish oil with blends of vegetable oils (rapeseed, linseed and palm oils) in diets for European sea bass (Dicentrarchus labrax L.) over a long term growth study: effects on muscle and liver fatty acid composition and effectiveness of a fish oil finishing diet. Comp. Biochem. Physiol., 145B: 389399. https://doi.org/10.1016/j.cbpb.2006.08.012

Mumoğullarında, P., 2012. The effect of alternative phases of canola oil and fish oil level on the growth performance and fatty acid profile of juvenile European sea bass (Dicentrarchus labrax). An MS thesis submitted to the Department of Aquaculture. Cukurova University, Adana, Turkey. pp. 63.

Nakamura, M.T. and Nara, T.Y., 2004. Structure, function, and dietary regulation of delta- 6 , delta- 5 , and delta- 9 
desaturases. Annu. Rev. Nutri., 24: 345-376. https:// doi.org/10.1146/annurev.nutr.24.121803.063211

Nandeesha, M.C., Gangadhara, B. and Manissery, J.K., 2002. Further studies on the use of mixed feding schedules with plant- and animal-based diets for common carp Cyprinus carpio (Linnaeus). Aquacul. Res., 33: 1157-1162. https://doi.org/10.1046/j.13652109.2002.00771.x

Ng, W-K., Sigholt, T. and Bell, J.G., 2004. The influence of environmental temperature on the apparent nutrient and fatty acid digestibility in Atlantic salmon (Salmo salar L.) fed finishing diets containing different blends of fish oil, rapeseed oil and palm oil. Aquacul. Res., 35: 1228-1237. https:// doi.org/10.1111/j.1365-2109.2004.01131.x

Ng, W-K., Chong, C-Y., Wang, Y. and Romano, N., 2013. Effects of dietary fish and vegetable oils on the growth, tissue fatty acid composition, oxidative stability and vitamin E content of red hybrid tilapia and efficacy of using fish oil finishing diets. Aquaculture, 372-375: 97-110. https://doi. org/10.1016/j.aquaculture.2012.10.030

NRC, 1993. Nutrient requirements of fish. National Academy Press. Washington DC, USA.

Regost, C., Arzel, J., Robin, J., Roselund, G. and Kaushik, S.J., 2003. Total replacement of fish oil by soybean or oil with return to fish oil in turbot (Psetta maxima) 1. Growth performance, flesh fatty acid profile, and lipid metabolism. Aquaculture, 217: 465-482. https://doi.org/10.1016/S0044-8486(02)00259-4

Robin, J.H., Regost, C., Arzel, J. and Kaushik, S.J., 2003. Fatty acid profile of fish following a change in dietary fatty acid source: model of fatty acid composition with a dilution hypothesis. Aquaculture, 225: 283293. https://doi.org/10.1016/S0044-8486(03)002965

Rosenlund, G., Obach, A., Gisvold, M., Standal, H. and Teit, K., 2001. Effect of alternative lipid sources on long term growth performance and quality of Atlantic salmon (Salmo salar). Aquacul. Res., 32 (Suppl. 1): 323-328. https://doi.org/10.1046/j.1355557x.2001.00025.x

Sargent, J.R., Tocher, D.R. and Bell, J.G., 2002. The lipids. In: Fish nutrition (eds. J.E. Halver and R.W. Hardy) third ed. Elsevier, USA, pp. 181-257. https:// doi.org/10.1016/B978-012319652-1/50005-7

Shahrooz, R., Agh, N., Jafari, N., Kalantari, A., Reza, J.A. and Karimi, A., 2018. Effects of fish oil replacement with vegetable oils in rainbow trout (Oncorhynchus mykiss) fingerlings diet on growth performance and foregut histology. Turkish J. Fish. aquat. Sci., 18: 825-832. https://doi.org/10.4194/1303- 2712-v18_6_09

Sprecher, H., Luthria, D.L., Mohammed, B.S. and Baykusheva, S.P., 1995. Are-evaluation of the pathways for the biosynthesis of polyunsaturated fatty acids. J. Lipid Res., 36: 2471-2477. https://doi. org/10.1201/9781439831939.ch2

Şener, E., 1996. Alabalık yetiştiriciliğinde kullanılan yemler ve yemlemenin önemi. Su Ürünleri Vakfi Dergisi, 4: 9-12.

Tacon, A., 2004. Use of fish meal and oil in aquaculture: A global perspective. Aquat. Resour. Cult. Develop., 1: 3-14.

Tocher, D.R., Fonseca-Madrigal, J., Bell, J.G., Dick, J.R., Henderson, R.J. and Sargent, J.R., 2002. Effects of diets containing linseed oil on fatty acid desaturation and oxidation in hepatocytes and intestinal enterocytes in Atlantic salmon (Salmo salar). Fish Physiol. Biochem., 26: 157-170.

Torstensen, B.E., Froyland, L. and Lie, O., 2004. Replacing dietary fish oil with increasing levels of rapeseed oil and olive oil: effects on Atlantic salmon (Salmo salar L.) tissue and lipoprotein composition and lipogenic enzyme activities. Aquacul. Nutr., 10: 175-192. https://doi.org/10.1111/j.13652095.2004.00289.x

Torstensen, B.E., Bell, J.G., Rosenlund, G., Henderson, R.J., Graff, I.E., Tocher, D.R., Lie, O. and Sargent, J.R., 2005. Tailoring of Atlantic salmon (Salmo salar L.) flesh lipid composition and sensory quality by replacing fish oil with a vegetable oil blend. $J$. agric. Fd. Chem., 53: 10166-10178. https://doi. org/10.1021/jf051308i

Trushenski, J.T. and Boesenberg, J., 2009. Influence of dietary fish oil concentration and finishing duration on beneficial fatty acid profile restoration in sunshine bass Morone chrysops $q \times \mathrm{M}$. saxatilis $\hat{\sigma}$. Aquaculture, 296: 277-283.

Turchini, G.M., Francis, D.S. and De Silva, S.S., 2006. Modification of tissue fatty acid composition in Murray cod (Maccullochella peeli peeli, Mitchell) resulting from a shift from vegetable oil diets to a fish oil diet. Aquacul. Res., 37: 570-585. https://doi. org/10.1111/j.1365-2109.2006.01465.x

Turchini, G.M., Francis, D.S. and De Silva, S.S., 2007. Finishing diets stimulate compensatory growth: results of a study on Murray cod, Maccullochella peelii peelii. Aquacul. Nutri., 13: 351-360. https:// doi.org/10.1111/j.1365-2095.2007.00483.x

Turchini, G.M. and Francis, D.S., 2009. Fatty acid metabolism (desaturation, elongation and betaoxidation) in rainbow trout fed fish oil- or linseed oil- based diets. Br. J. Nutri., 102: 69-81. https://doi. 
org/10.1017/S0007114508137874

Turchini, G.M., Francis, D.S., Senadheera, S.P.S.D., Thanuthong, T. and De Silva, S.S., 2011. Fish oil replacement with different vegetable oils in Murray cod: Evidence of an "omega-3 sparing effect" by other dietary fatty acids. Aquaculture, 315: 250-239. https://doi.org/10.1016/j.aquaculture.2011.02.016

Turchini, G.M., Moretti, V.M., Hermon, K., Caprino, F., Busetto, M.L., Bellagamba, F., Rankin, T., Keast, R.S. and Francis, D.S., 2013. Monola oil versus canola oil as a fish oil replacer in rainbow trout feeds: effects on growth, fatty acid metabolism and final eating quality. Fd. Chem., 141: 1335-1344. https://doi.org/10.1016/j.foodchem.2013.03.069
Yıldız, M., Eroldoğan, T.O., Ofori-Mensah, S., Engin, K. and Baltac1, M.A., 2018. The effects of fish oil replacement by vegetable oils on growth performance and fatty acid profile of rainbow trout: Re-feeding with fish oil finishing diet improved the fatty acid composition. Aquaculture, 488: 123-133. https://doi.org/10.1016/j.aquaculture.2017.12.030

Yilmaz, H.A., Corraze, G., Panserat, S. and Eroldoğan, O.T., 2016. Effects of alternate feeding with different lipid sources on fatty acid composition and bioconversion in European sea bass (Dicentrarchus labrax). Aquaculture, 464: 28-36. https://doi. org/10.1016/j.aquaculture.2016.06.013 